\title{
Clutter Loss Modelling for Low Elevation Link Scenarios
}

\author{
Pavel Valtr, ${ }^{1}$ Jan Zeleny, ${ }^{1}$ Pavel Pechac, ${ }^{1}$ and Martin Grabner ${ }^{2}$ \\ ${ }^{1}$ Department of Electromagnetic Field, Faculty of Electrical Engineering, Czech Technical University in Prague, \\ Technicka 2, 16627 Prague 6, Czech Republic \\ ${ }^{2}$ Czech Metrology Institute, Okruzni 31, 63800 Brno, Czech Republic \\ Correspondence should be addressed to Pavel Valtr; pavel.valtr@fel.cvut.cz
}

Received 26 January 2016; Revised 29 February 2016; Accepted 3 March 2016

Academic Editor: Giuseppe Mazzarella

Copyright (C) 2016 Pavel Valtr et al. This is an open access article distributed under the Creative Commons Attribution License, which permits unrestricted use, distribution, and reproduction in any medium, provided the original work is properly cited.

An analysis of the accuracy of several deterministic and semideterministic propagation models related to calculating clutter loss for low elevation links is presented. Predictions are compared with measurements carried out at $2 \mathrm{GHz}$ over three locations. By comparing prediction and measurement techniques, improved predictive capabilities of deterministic models are demonstrated and quantified in cases where a description of the propagation environment exists and an air-to-ground scenario is considered.

\section{Introduction}

Predicting the performance of communication systems requires unequivocal estimates of channel behavior, in particular, signal strength level at the receiver. The investigation of low elevation links has become increasingly important in the field of unmanned aerial vehicle (UAV) communication and holds great potential as UAVs can be directly applied in the military and civil sectors. Low elevation links are characterised by a transmitter location above surrounding obstacles and a receiver in close proximity to the ground. One of the crucial aspects of such links is loss due to shadowing by clutter, that is, buildings and trees surrounding the receiver.

Modelling clutter loss is frequently carried out using empirical or semideterministic models. The utilisation of empirical models is often dictated by the lack of precise local clutter data. Empirical models usually lack sufficient prediction accuracy but have the undisputed advantage of being unsophisticated and of requiring only few input parameters. Due to their nature, empirical models fit the measured data [1]; these models only produce approximate results, even in environments similar to those where the measurements were carried out. Physically based models, on the other hand, are apt to make more accurate predictions. However, they are substantially more complicated, such as Walfisch and Bertoni's model [2], when compared to empirical models.
Other approaches to model clutter and terrain diffraction loss utilise the physical optics method [3] leading to a numerical solution which is inherently demanding from a computational perspective. A reasonable trade-off between accuracy and model simplicity is needed to predict channel behavior especially in terms of the received signal level.

A clutter loss model is part of ITU Recommendation (ITU-R) P.452 [4] where additional diffraction loss of a terrestrial link due to buildings and vegetation is calculated. The model is a simple formula whose parameters must include a nominal obstacle height and distance as tabulated for the clutter categories. In a work by Maciel et al. [5], the authors presented simple formulas to calculate propagation over rooftops with a receiver at street level. Designed for groundto-ground propagation predictions where the transmitter is placed above rooftops, these semideterministic models are considered applicable to the air-to-ground propagation scenario also.

In this paper clutter loss predictions for low elevation angle geometries are analysed, together with other models, and compared with data obtained by measurements. The focal point of this work is to assess the suitability of utilising a single knife-edge model to predict clutter loss due to buildings where the transmitter elevation is higher when compared to conventional terrestrial transmitters, but lower than satellites, a scenario known as a low elevation link. 


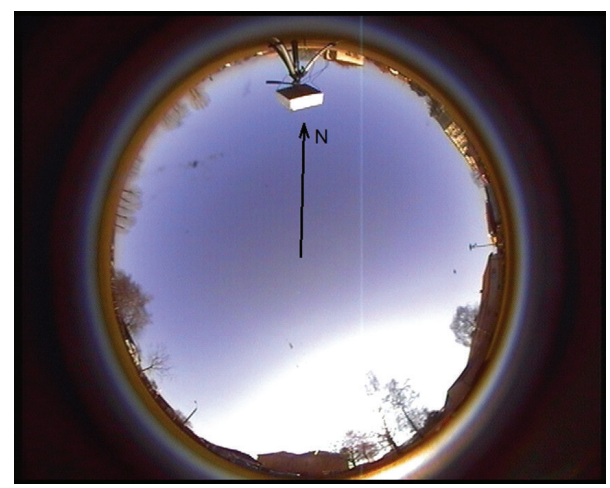

FIGURE 1: Scenario 1: the arrow indicates due north (0 deg. azimuth); the azimuth runs in a clockwise direction (the tripod with the receiving antenna was removed manually when the photo was processed).

\section{Measurement Scenarios and Set-Up}

A continuous-wave measurement was carried out at $2 \mathrm{GHz}$ with a transmitter attached to a helium filled remote controlled airship to achieve the desired elevation angles. The receiver was stationary and placed $1 \mathrm{~m}$ above the ground. A quarter-wave monopole antenna was used as both the transmitter and receiver to act as omnidirectional antennas for the measurement geometry considered. The received signal was sampled at a frequency of $10 \mathrm{k}$ samples per second. The sampled data were processed using moving average filter. The advantage of using relatively high sampling frequency is related to quantization error and its suppression. The airship, equipped with a GPS receiver to record its position, was flying in an approximate circle around the receiver with a radius of $1500 \mathrm{~m}$ and elevation of 4 degrees. One circle was flown for each scenario. Slight inaccuracies in path trajectory and elevation are accounted for in the models using actual GPS position data. The measurements were carried out in a village close to Prague, the Czech Republic, at three different locations.

The first scenario is represented by a relatively open space featuring a football pitch and some nearby buildings as can be seen in the fish-eye photograph in Figure 1. The second scenario is a narrow dead end street surrounded by buildings on three sides; see Figure 2. The third scenario is an open space with two high buildings nearby; see Figure 3.

\section{Propagation Models and Comparison with Measurements}

Fish-eye photographs were used to obtain an environmental description to determine the highest building elevation, representing the dominant obstacle, for each azimuth. A single knife-edge diffraction model was used to predict clutter loss attributable to these buildings; other objects, such as trees, were ignored. Figure 4 shows the geometry of the model used. The elevation and distance of the airship are ascertained by GPS. The distance of the dominant building obstacle from the receiver for each azimuth was taken from Google Earth.

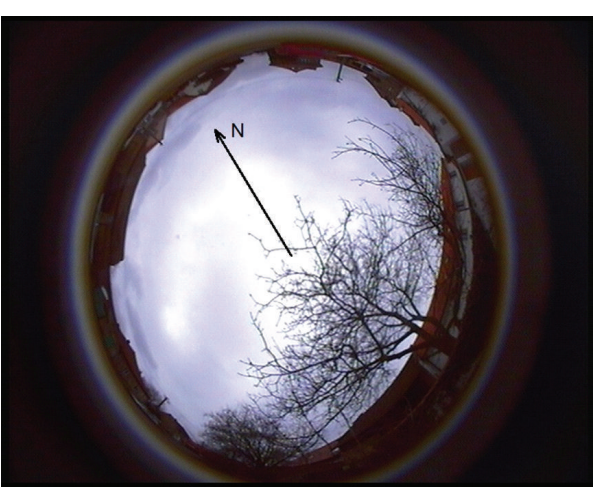

FIGURE 2: Scenario 2: the arrow indicates due north (0 deg. azimuth); the azimuth runs in a clockwise direction.

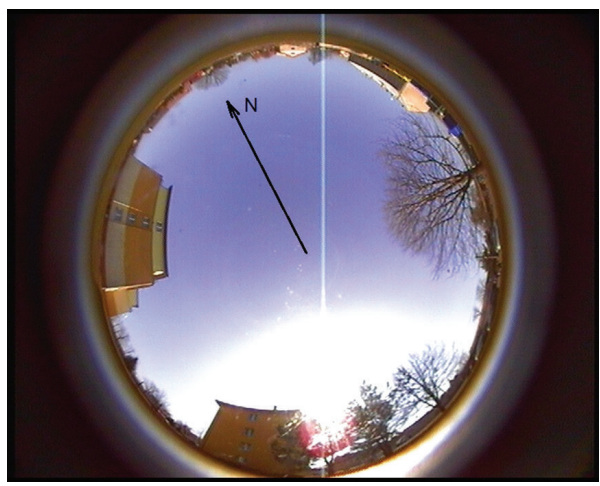

FIGURE 3: Scenario 3: the arrow indicates due north (0 deg. azimuth); the azimuth runs in a clockwise direction.

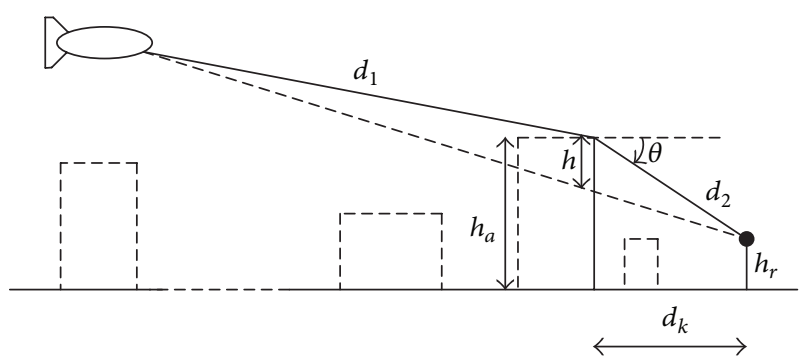

FIGURE 4: Model geometry.

Excess loss $L_{d}$, due to building diffraction, is calculated using the diffraction coefficient $v$ and the well-known formula [4]:

$$
L_{d}=6.9+20 \log _{10}\left(\sqrt{(v-0.1)^{2}+1}+v-0.1\right),
$$

where $L_{d}$ is set equal to zero for $v$ greater than -0.78 ; the diffraction coefficient $v$ is calculated as

$$
v=h \sqrt{\frac{2}{\lambda}\left(\frac{1}{d_{1}}+\frac{1}{d_{2}}\right)}
$$

where $\lambda$ is wavelength and where height $h$ and distances $d_{1}$ and $d_{2}$ are shown in Figure 4 . 


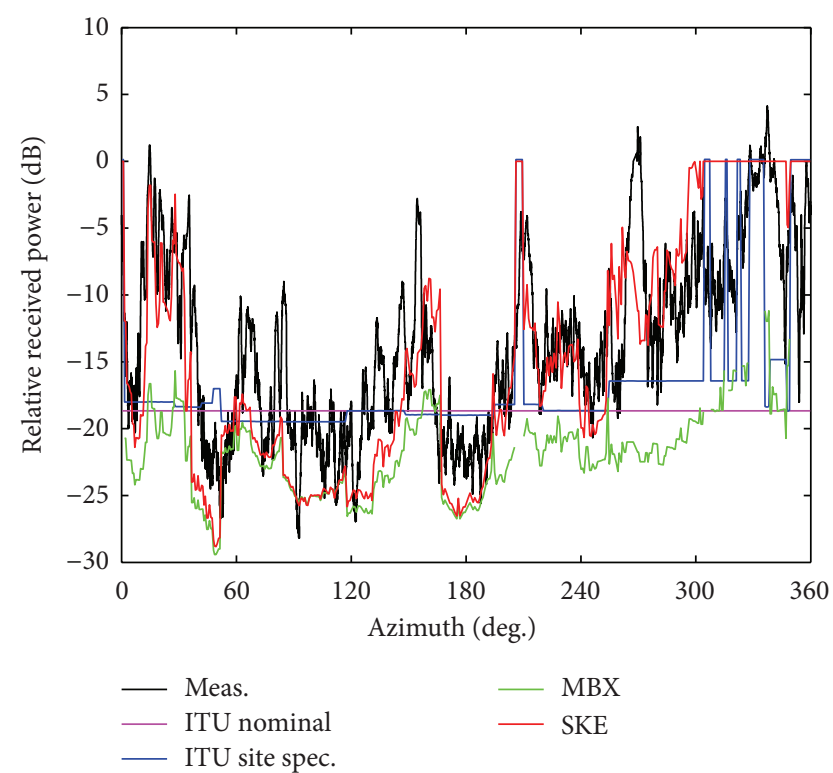

FIGURE 5: Scenario 1: a comparison of individual models: ITU-R P.452 nominal parameters (ITU nominal), ITU-R P.452 site-specific parameters (ITU site spec.), Maciel-Bertoni-Xia (MBX), single knife-edge (SKE), and measured data (meas.).

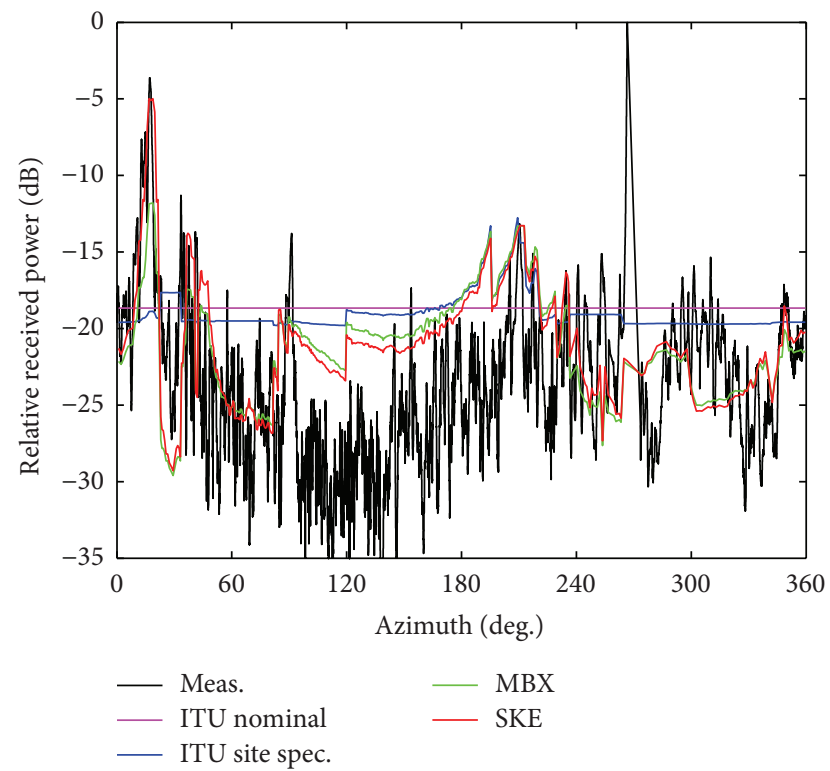

FIGURE 6: Scenario 2: a comparison of individual models: ITU-R P.452 nominal parameters (ITU nominal), ITU-R P.452 site-specific parameters (ITU site spec.), Maciel-Bertoni-Xia (MBX), Single knife-edge (SKE), and measured data (meas.).

Figures 5-7 show a comparison of the measured and predicted results for the three scenarios in terms of received power relative to free space as a function of their azimuths. The measured results are compared with the single knifeedge as discussed above. The comparison also includes the Maciel-Bertoni-Xia model [5, eq. (2)] and the clutter model

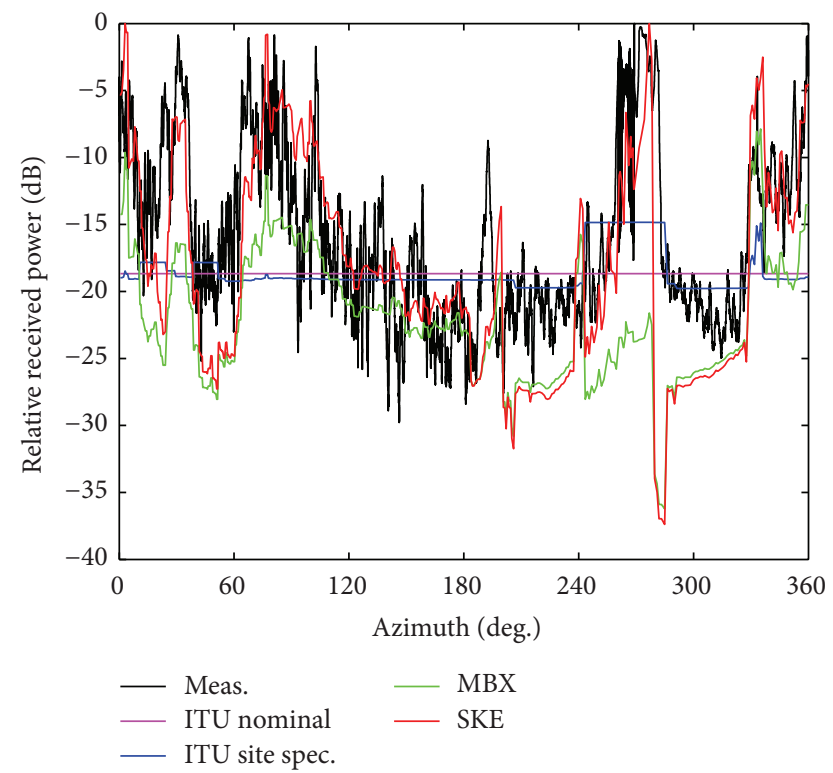

FIGURE 7: Scenario 3: a comparison of individual models: ITU-R P.452 nominal parameters (ITU nominal), ITU-R P.452 site-specific parameters (ITU site spec.), Maciel-Bertoni-Xia (MBX), Single knife-edge (SKE), and measured data (meas.).

from ITU-R P.452 [4]. Maciel-Bertoni-Xia model calculates loss due to shadowing by buildings as

$$
L=-10 \log _{10}\left[\frac{1}{\pi k \cdot d_{2}}-\left(\frac{1}{\theta}-\frac{1}{2 \pi+\theta}\right)^{2}\right],
$$

where $L$ is excess loss by building diffraction and $k$ is the wave number. Angle $\theta$ and distance $d_{2}$ are shown in Figure 4 .

ITU Recommendation ITU-R P.452-16 provides the following formula for additional clutter loss:

$$
\begin{aligned}
L_{c}= & 10.25 F_{f c} e^{-d_{k}}\left\{1-\tanh \left[6\left(\frac{h_{r}}{h_{a}}-0.625\right)\right]\right\} \\
& -0.33
\end{aligned}
$$

where $L_{c}$ is the clutter loss, $h_{r}$ is receiver height, $h_{a}$ is height of clutter above local ground level, and $d_{k}$ is distance (in $\mathrm{km}$ ) of receiver from the clutter; see Figure 4 . The frequency term $F_{f c}$ is calculated as

$$
F_{f c}=0.25+0.375\{1+\tanh [7.5(f-0.5)]\},
$$

where $f$ is frequency in $\mathrm{GHz}$.

Two implementations of the ITU model were used; the first utilises nominal parameters for a village center environment, in terms of obstacle height $h_{a}$ and distance $d_{k}$ as suggested in the recommendation. The other uses site-specific parameters obtained from fish-eye photographs and a digital map. It can be observed that the ITU-R model using nominal parameters gives a rudimentary estimate of clutter loss and is only suitable when there are no environmental data available. Including site-specific data in the ITU model improves the predictive capabilities of the model slightly. The MacielBertoni-Xia model matches measurements for points of high 
TABLE 1: Root mean square error (dB).

\begin{tabular}{lcccc}
\hline Scenario/model & Maciel-Bertoni-Xia & ITU, nominal param. & ITU, site-specific param. & Single knife-edge \\
\hline Scenario 1 & 8.8 & 8.8 & 7.1 & 5.7 \\
Scenario 2 & 5.4 & 8.0 & 7.4 & 5.2 \\
Scenario 3 & 8.4 & 7.2 & 7.0 & 6.4 \\
\hline
\end{tabular}

loss only. This model fails to provide accurate results in, or close to, line-of-sight situations. The single knife-edge model is the most accurate one of all the models analysed for the given low elevation link scenario. The inaccuracies of the ITU and Maciel-Bertoni-Xia model reside in the fact that only the position of the receiver with respect to the obstacle is considered while ignoring the elevation and distance of the transmitter.

The accuracy of the four models mentioned was analysed using root mean square error (RMSE) and calculated as

$$
\mathrm{RMSE}=\sqrt{\frac{1}{N} \sum_{i=1}^{N}\left(P_{p i}-P_{m i}\right)^{2}},
$$

where $P_{p i}$ and $P_{m i}$ are predicted and measured relative received power in $\mathrm{dB}$, respectively. Values of the root mean square error for all methods and all three scenarios are shown in Table 1 . The values obtained show good prediction accuracy in terms of root mean square error when compared to other models for path loss prediction where reported RMSE is in the order of $12-15 \mathrm{~dB}$ at best [6].

\section{Conclusion}

The importance of site-specific geometry, including the elevation angle in clutter modelling for low elevation links, was demonstrated. While the complexity of all models analysed is comparable, the single knife-edge model leads to highly accurate predictions of clutter loss as long as a description of the propagation environment is available in terms of obstacle height and location as demonstrated in several low elevation scenarios.

\section{Competing Interests}

The authors declare that they have no competing interests.

\section{Acknowledgments}

This work was supported by Czech Science Foundation Grant no. P102/14-01527S, "Basic Research of Propagation of Electromagnetic Waves in the Atmospheric Boundary Layer for Low Elevation Links."

\section{References}

[1] M. Hata, "Empirical formula for propagation loss in land mobile radio services," IEEE Transactions on Vehicular Technology, vol. 29, no. 3, pp. 317-325, 1980.
[2] J. Walfisch and H. L. Bertoni, "A Theoretical Model of UHF Propagation in Urban Environments," IEEE Transactions on Antennas and Propagation, vol. 36, no. 12, pp. 1788-1796, 1988.

[3] J. H. Whitteker, "Physical optics and field-strength predictions for wireless systems," IEEE Journal on Selected Areas in Communications, vol. 20, no. 3, pp. 515-522, 2002.

[4] "Prediction procedure for the evaluation of interference between stations on the surface of the Earth at frequencies above about 0.1 GHz,' Tech. Rep. P.452-16, ITU, Geneva, Switzerland, 2015.

[5] L. R. Maciel, H. L. Bertoni, and H. H. Xia, "Unified approach to prediction of propagation over buildings for all ranges of base station antenna height," IEEE Transactions on Vehicular Technology, vol. 42, no. 1, pp. 41-45, 1993.

[6] C. Phillips, D. Sicker, and D. Grunwald, "Bounding the practical error of path loss models," International Journal of Antennas and Propagation, vol. 2012, Article ID 754158, 21 pages, 2012. 


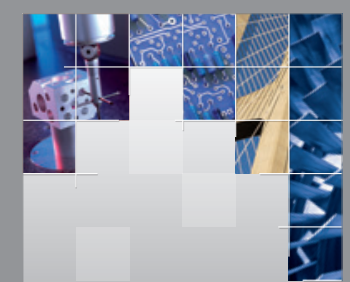

\section{Enfincering}
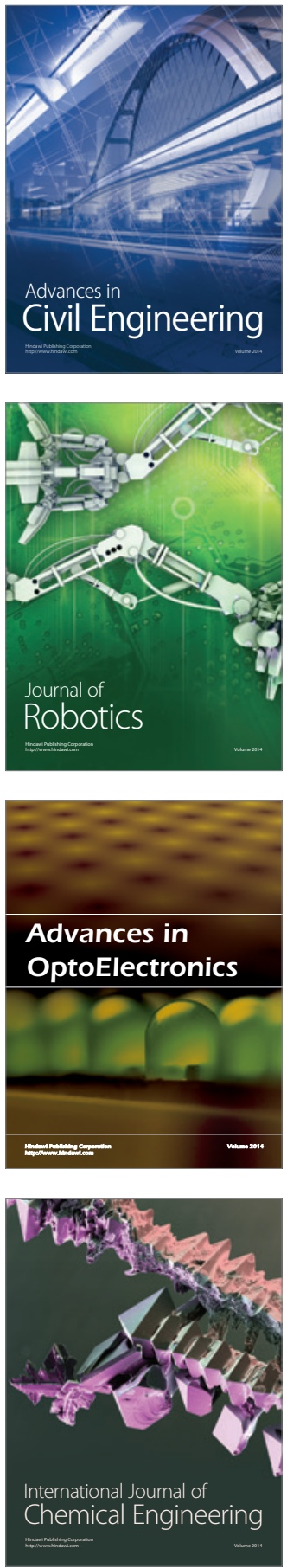

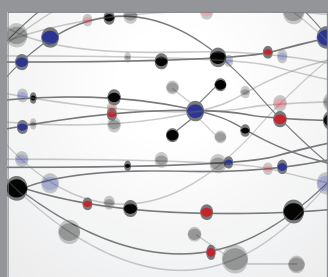

The Scientific World Journal

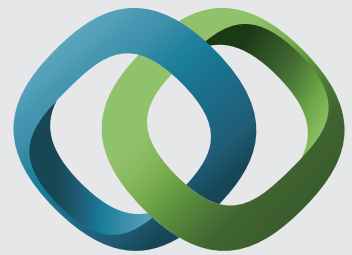

\section{Hindawi}

Submit your manuscripts at

http://www.hindawi.com
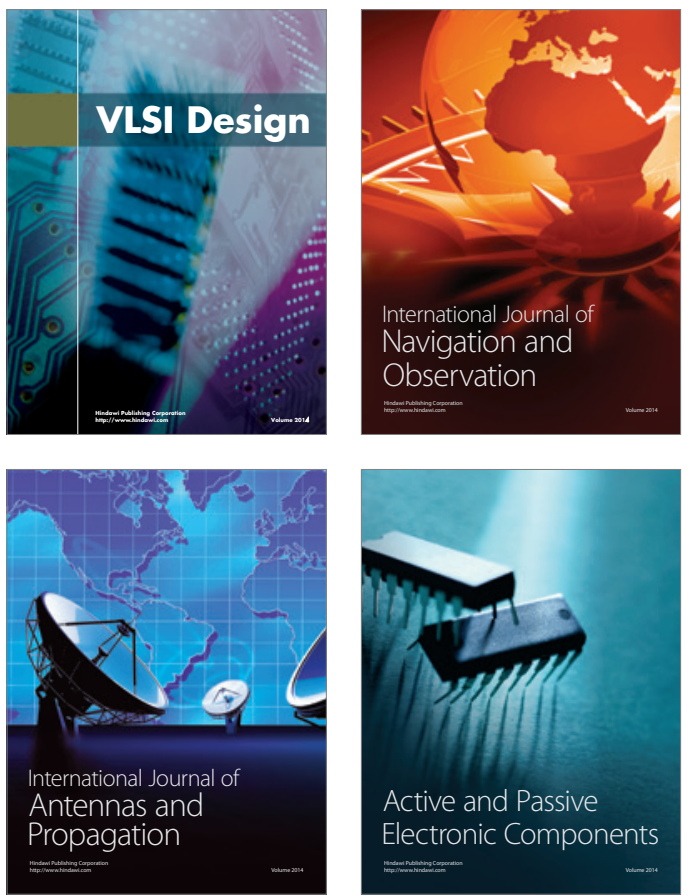
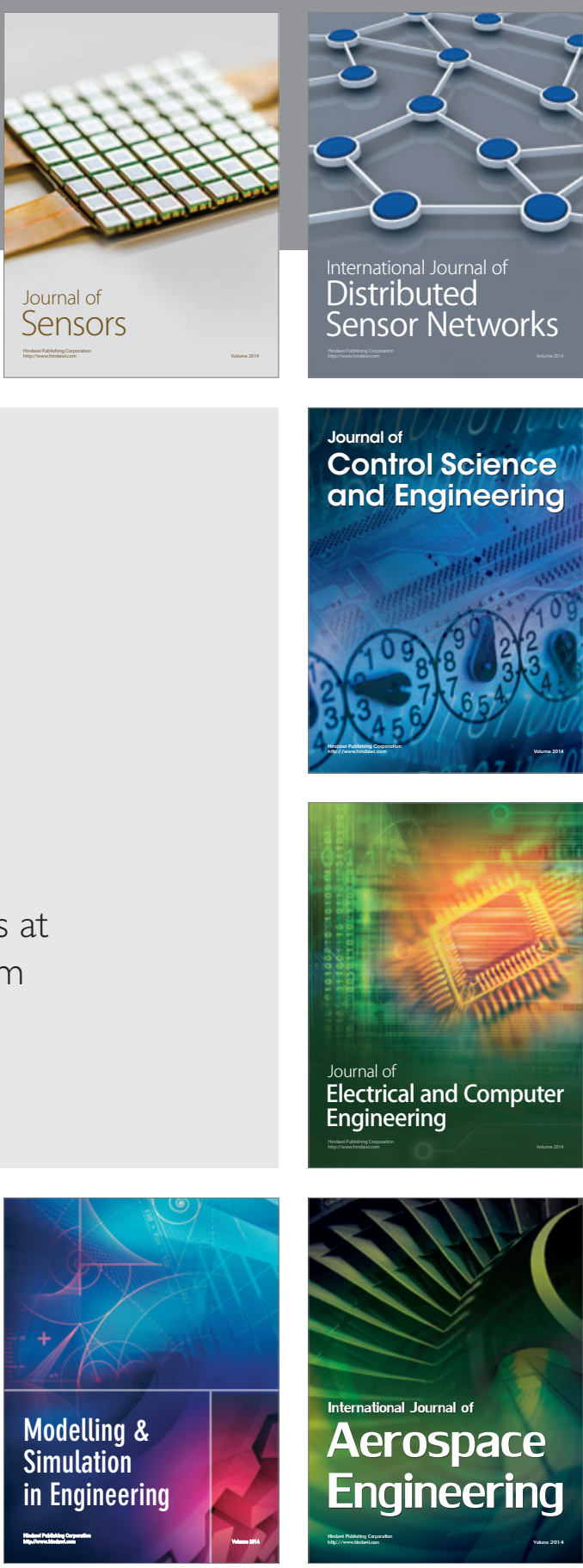

International Journal of

Distributed

Sensor Networks

Journal of

Control Science

and Engineering
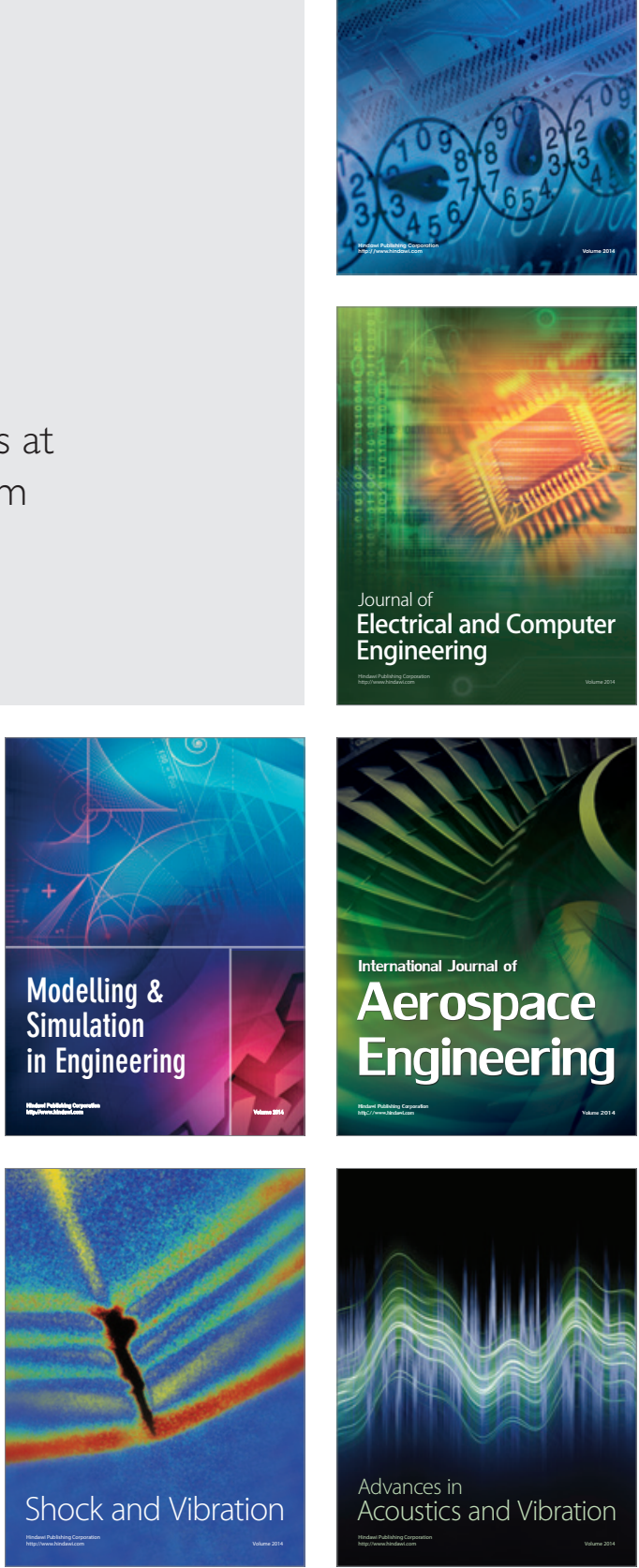